\title{
26. SHORE LABORATORY REPORT ON MESOZOIC FORAMINIFERIDA, LEG 17
}

\author{
Emile A. Pessagno, Jr. and Jose F. Longoria, Geosciences Division, \\ University of Texas at Dallas, Dallas, Texas
}

$165 \mathrm{~A}-17-1,120-122 \mathrm{~cm}$

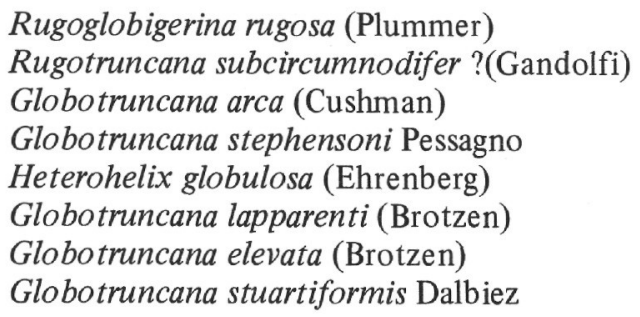

Rugoglobigerina rugosa (Plummer)

Rugotruncana subcircumnodifer?(Gandolfi)

Globotruncana arca (Cushman)

Globotruncana stephensoni Pessagno

Heterohelix globulosa (Ehrenberg)

Globotruncana lapparenti (Brotzen)

Globotruncana elevata (Brotzen)

Globotruncana stuartiformis Dalbiez

Biostratigraphic determination: G. fornicata-stuartiformis Assemblage-zone, $G$. elevata Subzone to $R$. subcircumnodifer Subzone; late Campanian to early Maastrichtian, sparse fauna.

\section{$167-50-1,80-82 \mathrm{~cm}$}

Globotruncana fornicata (Plummer) Globotruncana subspinosa Pessagno Globotruncanella havanensis (Voorwijk)

Globigerinelloides volutus (White)

Heterohelix globulosus (Ehrenberg) Heterohelix sp.

Biostratigraphic determination: G. fornicata-stuartiformis Zone: $G$. elevata-R. subcircumnodifer Subzone, late Campanian-early Maastrichtian.

\section{$167-16-6$}

Globotruncana hilli Pessagno

Heterohelix globulosus (Ehrenberg)

Globigerinelloides sp. cf. prairiehillensis Pessagno

Globotruncanella sp.

Biostratigraphic determination: Same as above.

\section{$167-42-1,143-145 \mathrm{~cm}$}

Globotruncana plummerae Gandolfi
Globotruncana bulloides (Vogler)
Globotruncana stephensoni Pessagno
Globotruncana elevata (Brotzen)
Globotruncana nothi Bronnimann and Brown
Globotruncana stuartiformis Dalbiez
Globotruncana fornicata (Plummer)
Pseudoguembelina costulata (Cushman)

Biostratigraphic determination: G. fornicata-stuartiformis Assemblage-Zone, G. elevata Subzone ( $G$. calcarata Zonule) to $R$. subcircumnodifer Subzone; late Campanian to early Maastrichtian.
$167-42-4,40-42 \mathrm{~cm}$

Globotruncana arca (Cushman)

Globotruncana elevata (Brotzen)

Rugoglobigerina rugosa Plummer

Rugotruncana subcircumnodifer (Gandolfi)

Globigerinelloides prairiehillensis Pessagno

Globigerinelloides globulosus (Ehrenberg)

Pseudoguembelina costulata (Cushman)

Heterohelix punctulatus (Cushman)

Globotruncana fornicata (Plummer)

Globotruncana hilli Pessagno

Pseudotextularia elegans (Rzehak)

Globotruncana stuartiformis (Dalbiez

Globotruncana sp. cf. Globotruncana nothi (Bronnimann and Brown)

Globotruncana plummerae Gandolfi

Biostratigraphic determination: $G$. fornicata-stuartiformis Assemblage-zone, $G$. elevata Subzone to $R$. subcircumnodifer Subzone; late Campanian to early Maastrichtian.

\section{$167-43-2,10-12 \mathrm{~cm}$}

Quaternary contaminants (Very abundant)
Globotruncana arabica El-Naggar
Globotruncana fornicata Plummer
Globotruncana linneiana (d'Orbigny)
Globotruncana hilli Pessagno
Globotruncana plummerae Gandolfi
Globotruncana gansseri Bolli (rare)
Globotruncana aegyptiaca Nakkady
Globotruncana elevata Brotzen
Globotruncana stuartiformis Dalbiez
Globigerinelloides prairiehillensis Pessagno
Rugoglobigerina rugosa Plummer
Rugoglobigerina scotti (Bronnimann)
Rugoglobigerina hexacamerata Bronnimann
Pseudoguembelina costulata (Cushman)
Globotruncanella mattsoni (Pessagno)

Biostratigraphic determination: $G$. fornicata-stuartiformis Assemblage-Zone, $R$. subcircumnodifer ( $R$. subpennyi Zonule); early Maastrichtian.

\section{$167-54-1,126-128 \mathrm{~cm}$}

Globotruncana fornicata Plummer Globotruncana lapparenti (Brotzen) Globotruncana stuartiformis Dalbiez Quaternary planktonic foraminifera (very abundant) 
Biostratigraphic determination: Globotruncana fornicatastuartiformis Assemblage-Zone.

\section{$167-44-1$}

Rugoglobigerina sp.

$171-9-3,80-82 \mathrm{~cm}$

Rugoglobigerina rotundata (Bronnimann)

Heterohelix globulosus (Ehrenberg)

Heterohelix sp

Pseudoguembelina excolata (Cushman)

Tertiary foraminifera

Biostratigraphic determination: G. contusa-stuartiformis Assemblage-Zone; late Maastrichtian.

$171-10-4,30-32 \mathrm{~cm}$

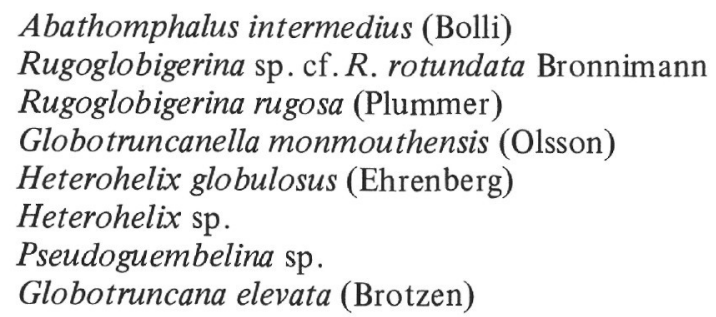

Biostratigraphic determination: G. contusa-stuartiformis Assemblage-Zone, Abathomphalus mayaroensis Subzone; late Maastrichtian.

$171-10-5,30-32 \mathrm{~cm}$

Globotruncanella havanensis (Voorwijk)

Rugoglobigerina scotti (Bronnimann)

Rugoglobigerina rugosa (Plummer)

Pseudoguembelina excolata (Cushman)

Biostratigraphic determination: G. contusa-stuartiformis Assemblage-Zone, A. mayaroensis Subzone; late Maastrichtian via superposition.

\section{$171-11-4,30-32 \mathrm{~cm}$}

Rugoglobigerina hexacamarata Bronnimann

Rugoglobigerina rugosa (Plummer)

Globotruncanella havanensis (Voorwijk)

Pseudoguembelina excolata (Cushman)

Pseudoguembelina costulata (Cushman)

Biostratigraphic determination: Same as above.

$171-11-5,30-32 \mathrm{~cm}$

\footnotetext{
Abathomphalus intermedius (Bolli)

Globotruncana gansseri (Bolli)

Globotruncana aegyptiaca Nakkady

Globotruncana contusa (Cushman)

Globotruncana arca (Cushman)

Rugoglobigerina rugosa (Plummer)

Rugoglobigerina sp.
}

\begin{abstract}
Heterohelix globulosus (Ehrenberg) Heterohelix punctulatus (Cushman) Heterohelix striatus (Ehrenberg)

Pseudoguembelina costulata (Cushman)

Pseudoguembelina excolata (Cushman)
\end{abstract}

Biostratigraphic determination: G. contusa-stuartiformis Assemblage-Zone, G. gansseri Subzone; late Maastrichtian.

\section{$171-13-5,30-32 \mathrm{~cm}$}

Globotruncanella havanensis (Voorijk)

Globotruncanella monmouthensis (Olsson)

Rugoglobigerina macrocephala Bronnimann

Globotruncana fornicata Plummer

Globotruncana arca (Cushman)

Globotruncana trinidadensis Gandolfi

Globotruncana stephensoni Pessagno

Globotruncana elevata Brotzen

Globotruncana bulloides (Vogler)

Globotruncana hilli Pessagno

Globotruncana trinidadensis Gandolfi

Globigerinelloides yaucoensis (Pessagno)

Globotruncana prairiehillensis Pessagno

Heterohelix punctulatus (Cushman)

Pseudoguembelina excolata (Cushman)

Rugoglobigerina rugosa (Plummer)

Rugoglobigerina sp.

Biostratigraphic determination: G. fornicata-stuartiformis Assemblage-Zone, $R$. subcircumnodifer Subzone, top of $R$. subpennyi Zonule.

171-15-1-3-4-5, 30-32 cm

Globotruncana stephensoni Pessagno

Globotruncana arca (Cushman)

Globotruncana stuartiformis Dalbiez

Rugoglobigerina rugosa (Plummer)

Globotruncanella cf. G. havanensis (Voorwijk)

Heterohelix punctulatus Cushman

Biostratigraphic determination: Same as above via superposition.

\section{$171-16-1-3-4-5,40-42 \mathrm{~cm}$}

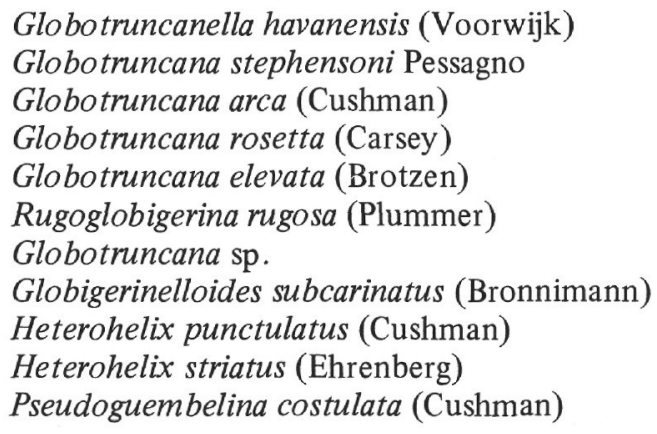

Biostratigraphic determination: G. fornicata-stuartiformis Assemblage-Zone, $R$. subpennyi Subzone, early Maastrichtian. 
$171-16-5,30-32 \mathrm{~cm}$

Globotruncana arca (Cushman)

Globotruncana fornicata Plummer

Globotruncana bulloides (Vogler)

Globotruncana stuartiformis Dalbiez

Globotruncana elevata (Brotzen)

Globotruncana nothi Bronnimann and Brown

Rugotruncana subcircumnodifer (Gandolfi)

Rugotruncana subpennyi Bronnimann

Globotruncana stephensoni Pessagno

Biostratigraphic determination: G. fornicata-stuartiformis Assemblage-Zone, $R$. subcircumnodifer Subzone, $R$. subpennyi Zonule.

\section{$171-17-2,30-32 \mathrm{~cm}$}

Globotruncana gansseri Bolli

Globotruncana lapparenti (Brotzen)

Globotruncana elevata (Brotzen)

Globotruncana plummerae Gandolfi

Globotruncana arca (Cushman)

Globotruncana stephensoni Pessagno

Globotruncana rosetta (Carsey)

Heterohelix punctulatus (Cushman)

Pseudoguembelina excolata (Cushman)

Pseudoguembelina costulata (Cushman)

Rugoglobigerina rugosa (Plummer)

Rugoglobigerina sp.

Rugotruncana subpennyi (Gandolfi)

Globigerinelloides prairiehillensis Pessagno

Globigerinelloides sp. cf. Globigerinelloides subcarinatus (Bronnimann)

Globotruncanella havanensis (Voowijk)

Biostratigraphic determination: G. fornicata-stuartiformis Assemblage-Zone, $R$. subcircumnodifer Subzone, top of $R$. subpennyi Zonule, early Maastrichtian

$171-17-3,30-32 \mathrm{~cm}$

Globotruncana gansseri Bolli

Globotruncana aegyptiaca Nakkady

Globotruncana fornicata Plummer

Globotruncana stuartiformis Dalbiez

Globotruncana elevata Brotzen

Globotruncana stephensoni Pessagno

Globotruncana arca (Cushman)

Globotruncana bulloides (Vogler)

Rugotruncana subpennyi (Gandolfi)

Rugotruncana subcircumnodifer (Gandolfi)

Pseudoguembelina excolata (Cushman)

Heterohelix punctulatus (Cushman)

Heterohelix sp.

Biostratigraphic determination: G. fornicata-stuartiformis Assemblage-Zone, top of $R$. subcircumnodifer Subzone, top of $R$. subpennyi Zonule.

$171-17-4,50-52 \mathrm{~cm}$

Globotruncanella havanensis (Voorwijk)

Globotruncana gansseri Bolli
Globotruncana aegyptiaca Nakkady

Globotruncana stephensoni Pessagno

Globotruncana elevata Brotzen

Globotruncana stuartiformis Dalbiez

Globotruncana plummerae Gandolfi

Globotruncana fornicata Cushman

Rugotruncana subcircumnodifer (Gandolfi)

Globotruncana lapparenti (Brotzen)

Globigerinelloides prairiehillensis Pessagno

Globigerinelloides yaucoensis (Pessagno)

Pseudoguembelina costulata (Cushman)

Heterohelix punctulatus (Cushman)

Biostratigraphic determination: G. fornicata-stuartiformis Assemblage-Zone, top of $R$. subcircumnodifer Subzone, top of $R$. subpennyi Zonule.

\section{$171-17-5,30-32 \mathrm{~cm}$}

Globotruncana fornicata Plummer

Globotruncana aegyptiaca Nakkady

Globotruncana plummerae Gandolfi

Globotruncana gansseri Bolli

Globotruncana elevata (Brotzen)

Globotruncana stuartiformis Dalbiez

Globotruncana lapparenti Brotzen

Heterhelix punctulatus (Cushman)

Biostratigraphic determination: G. fornicata-stuartiformis Assemblage-Zone, top of $R$. subcircumnodifer Subzone, top of $R$. subpennyi Zonule.

\section{$171-17-6,30-32 \mathrm{~cm}$}

Globotruncana aegyptiaca Nakkady

Globotruncana plummerae Gandolfi

Globotruncana contusa (Cushman)

Globotruncana arca (Cushman)

Globotruncana stephensoni Pessagno

Globotruncana fornicata Plummer

Globotruncana stuartiformis Dalbiez

Globotruncana elevata Brotzen

Rugotruncana cf. subpennyi (Gandolfi)

Rugoglobigerina rugosa (Plummer)

Globigerinelloides prairiehillensis Pessagno

Globigerinelloides sp. cf. Globigerinelloides subcarinatus

Globigerinelloides sp.

Heterohelix punctulatus (Cushman)

Heterohelix sp.

Biostratigraphic determination: G. fornicata-stuartiformis, top of $R$. subcircumnodifer Subzone, R. subpennyi Zonule.

$171-19-1,30-32 \mathrm{~cm}$

Globotruncana fornicata Plummer

Globotruncana aegyptiaca Nakkady

Globotruncana gansseri Bolli

Globotruncana elevata (Brotzen)

Globotruncana linneiana (d'Orbigny)

Globotruncana arca (Cushman)

Globotruncana rosetta (Carsey)

Globotruncana stephensoni Pessagno 
Heterohelix punctulatus (Cushman)

Heterohelix sp.

Rugoglobigerina macrocephala Bronnimann

Biostratigraphic determination: $G$. fornicata-stuartiformis Assemblage-Zone, top of $R$. subcircumnodifer Subzone, top of $R$. subpennyi Zonule, early Maastrichtian.

\section{$171-19-3,30-32 \mathrm{~cm}$}

Globotruncanella havanensis (Voorwijk)

Globotruncana petaloidea (Gandolfi)

Globotruncana mattsoni (Pessagno)

Globotruncana sp.

Globotruncana arca (Cushman)

Globotruncana stephensoni Pessagno

Globotruncana plummerae Gandolfi

Globotruncana linneiana (d'Orbigny)

Globotruncana rosetta (Carsey)

Globotruncana elevata (Brotzen)

Pseudoguembelina sp. cf. R. costulata (Cushman)

Heterohelix striatus (Ehrenberg)

Heterohelix punctulatus (Cushman)

Heterohelix cf. pulchra (Brotzen)

Biostratigraphic determination: G. fornicata-stuartiformis Assemblage-Zone, $R$. subcircumnodifer Subzone, top of $R$. subpennyi Zonule

\section{$171-22-2,30-32 \mathrm{~cm}$}

Globotruncana (?) fornicata Plummer

Marginotruncana angusticarenata Gandolfi

Marginotruncana coronata (Bolli)

Marginotruncana renzi (Gandolfi)

Whiteinella archaeocretacea Pessagno

Heterohelix reussi (Cushman)

Biostratigraphic determination: Marginotruncana concavata Subzone, early Santonian.

\section{$171-23-4,62-64 \mathrm{~cm}$}

\author{
Marginotruncana renzi (Gandolfi) \\ Marginotruncana coronata (Bolli) \\ Marginotruncana angusticarenata (Gandolfi) \\ Marginotruncana marginata (Reiss) \\ Whiteinella archaeocretacea Pessagno \\ Marginotruncana imbricata (Mornod) \\ Marginotruncana difformis (Gandolfi) \\ Heterohelix reussi (Cushman)
}

Biostratigraphic determination: Marginotruncana renzi Assemblage-Zone, "Coniacian."

\section{$171-23-5,30-32 \mathrm{~cm}$}

Marginotruncana indica (Jacbo and Sastry)

Marginotruncana coronata (Bolli)
Marginotruncana angusticamerata (Gandolfi)

Marginotruncana renzi (Gandolfi)

Whiteinella archaeocretacea Pessagno

Heterohelix reussi (Cushman)

Biostratigraphic determination: Marginotruncana renzi Assemblage-Zone, "Coniacian."

\section{$171-24-1,48-50 \mathrm{~cm}$}

$$
\begin{aligned}
& \text { Marginotruncana difformis (Gandolfi) } \\
& \text { Marginotruncana coronata (Bolli) } \\
& \text { Marginotruncana renzi (Gandolfi) } \\
& \text { Whiteinella archaeocretacea Pessagno }
\end{aligned}
$$

Biostratigraphic determination: M. renzi Assemblage-Zone, Coniacian.

\section{$171-25-3-4-5$}

$$
\begin{aligned}
& \text { Marginotruncana renzi (Gandolfi) } \\
& \text { Marginotruncana coronata (Bolli) } \\
& \text { Marginotruncana canaliculata (Reuss) } \\
& \text { Marginotruncana difformis (Gandolfi) } \\
& \text { Marginotruncana indica (Jacob and Sastry) } \\
& \text { Whiteinella archaeocretacea Pessagno } \\
& \text { Heterohelix sp. }
\end{aligned}
$$

Biostratigraphic determination: Marginotruncana renzi Assemblage-Zone, Conianian.

\section{$171-26-1,97-98 \mathrm{~cm}$}

$$
\begin{aligned}
& \text { Marginotruncana renzi (Gandolfi) } \\
& \text { Marginotruncana helvetica (Bolli) } \\
& \text { Marginotruncana coronata (Bolli) } \\
& \text { Marginotruncana angusticarenata (Gandolfi) } \\
& \text { Marginotruncana sigali (Reichel) } \\
& \text { Marginotruncana indica Jacob and Sastry) } \\
& \text { Marginotruncana canaliculata (Reuss) } \\
& \text { Whiteinella archaeocretacea Pessagno } \\
& \text { Heterohelix reussi (Cushman) }
\end{aligned}
$$

Biostratigraphic determination: Marginotruncana helvetica Assemblage-Zone, Whiteinella archaeocretacea Subzone; late Turonian.

\section{$171-26-2,30-32 \mathrm{~cm}$}

$$
\begin{aligned}
& \text { Marginotruncana sigali (Reichel) } \\
& \text { Marginotruncana angusticarenata (Gandolfi) } \\
& \text { Marginotruncana canaliculata (Reuss) } \\
& \text { Marginotruncana difformis (Gandolfi) }
\end{aligned}
$$

Biostratigraphic determination: Marginotruncana helvetica Assemblage-Zone, Whiteinella archaeocretacea Subzone, late Turonian. 